\title{
Inhaltsverzeichnis
}

Anne Bohnenkamp

Einleitung ......

Ulrike Landfester

Ein offenes Buch?

Autorschaft im Zeitalter der Informationsgesellschaft

Rüdiger Nutt-Kofoth

Sichten - Perspektiven auf Text

Peter Stadler

Die Grenzen meiner Textverarbeitung

bedeuten die Grenzen meiner Edition.

Charlotte Wahl

Leibniz' mathematische Notation und der Druck 41

Karin Vorderstemann

Medienwechsel als Mittel der Popularisierung:

Ziglers Asiatische Banise von 1689 bis heute 61

Winfried Woesler

Schillers Bühnenfassungen der Jungfrau von Orleans

im Vergleich zur Buchfassung.....

Ariane Martin, Dagmar von Hoff

Edition als Medientransfer, Edition im Medientransfer

Überlegungen am Beispiel von Büchners Woyzeck. 81

Jürgen Hein

Spieltexte des Wiener Volkstheaters:

Von der Handschrift zum „Manuskriptdruck“ 


\section{Wolfgang Lukas}

Medienwechsel und produktionsästhetische Logik:

Zu Paul Wührs O-Ton-Hörspiel So eine Freiheit

Toni Bernhart

Audioedition. Auf dem Weg zu einer Theorie

Reinmar Emans

Neue Darstellungsformen von Fassungen musikalischer Werke

Johannes Kepper, Christine Siegert

Oper multimedial - Zur Edirom-Ausgabe

von Haydns Arienbearbeitungen

\section{Iacopo Cividini}

$\mathrm{Zu}$ einer digitalen Edition des Operntextes von Mozarts

Idomeneo oder: Wie authentisch darf Mozart sein?

Alexander Becker, Christopher Grafschmidt, Stefan König, Stefanie Steiner

Möglichkeiten und Konsequenzen der Digitalen

Musikedition am Beispiel der Reger-Werkausgabe (RWA)

\section{Pascale Sutter}

Werkstattbericht: Die Retrodigitalisierung

der Sammlung Schweizerischer Rechtsquellen (SSRQ)

und die künftige digitale Edition

Simone Schultz-Balluff, Stefanie Dipper

St. Anselmi Fragen an Maria - Schritte zu einer

(digitalen) Erschließung, Auswertung und Edition

der gesamten deutschsprachigen Überlieferung (14.-16. Jh.)

Andrea Hofmeister-Winter

Alte Bits in neuen Codes: Gedanken zur Weiterentwicklung der ,Grazer dynamischen Editionsmethode' am Beispiel eines Editionsprojektes in Fortsetzungen.

Claudia Nitschke, Yvonne Pietsch

Elektronische Palimpseste:

Hybridedition von Ludwig Achim von Arnims Wintergarten. 207 


\section{Charlotte Cailliau}

Medienwechsel in der Praxis:

Die Verzen (1894) von Willem Kloos.

Ulrike Steierwald, Uta Störl, Hartmut Vinçon,

unter Mitarbeit von Stefan Gründling

Die Online-Edition der Briefe von und an Frank Wedekind.

Eine virtuelle Kontextualisierung von Korrespondenzen

Stephanie Jordans

Gedrucktes Buch und digitales Archiv.

Zur textkritischen und kommentierten Ausgabe

von Ernst Meisters lyrischem Werk

Bodo Plachta

Schreibtische

Anschriften 
